\title{
Combining vestibular rehabilitation with noisy galvanic vestibular stimulation for treatment of bilateral vestibulopathy
}

\author{
Josefine Eder $^{1} \cdot$ Silvy Kellerer $^{1} \cdot$ Tamara Amberger $^{1,2} \cdot$ Aram Keywan $^{1} \cdot$ Julia Dlugaiczyk $^{1,3} \cdot$ Max Wuehr $^{1}$. \\ Klaus Jahn ${ }^{1,2}$ (1)
}

Received: 29 October 2021 / Revised: 10 February 2022 / Accepted: 15 February 2022 / Published online: 25 February 2022

(c) The Author(s) 2022

\begin{abstract}
Objective Noisy galvanic vestibular stimulation (nGVS) has been shown to partly restore vestibular function and to stabilize stance and gait in patients with incomplete bilateral vestibulopathy (BVP). Here, we examined potential synergistic effects of nGVS when combined with standardized vestibular rehabilitation training (VRT).

Methods 23 patients with confirmed BVP received a 30-min vestibular rehabilitation training (VRT) program three times a week for 2 weeks. The intervention group $(n=12)$ was stimulated with nGVS (at individually determined optimal amplitudes) during training, whereas the control group $(n=11)$ received zero-amplitude nGVS (sham stimulation) during training. Outcome measurements assessed at baseline, after 2 weeks of training, and at 2-week follow-up included quantitative posturography, instrumented gait analysis, Timed Up and Go Test (TUG), Functional Gait Assessment (FGA), and clinical scores related to quality of life and balance confidence.

Results After 2 weeks of VRT, all patients showed moderate improvement in balance. Irrespective of nGVS treatment, performance improved in the TUG $(p<0.013)$, and in the FGA $(p<0.040)$. Furthermore, base of support when walking with closed eyes was reduced after 2 -week training $(p<0.003)$. Postural sway did not change. There was no difference between groups and thereby no evidence for an additional influence of nGVS on the VRT treatment effects.

Conclusion nGVS does not induce synergistic treatment effects in combination with VRT in patients with BVP when applied during treatment sessions. Hence, rather than being applied in parallel, nGVS and VRT might be complementary therapeutic options with nGVS being used during postural activities in daily life, e.g., walking.
\end{abstract}

Keywords Bilateral vestibulopathy $\cdot$ Vestibular rehabilitation $\cdot$ Noisy galvanic vestibular stimulation $\cdot$ Balance $\cdot$ Gait

\section{Introduction}

Patients with bilateral vestibulopathy (BVP) suffer from a complete or incomplete loss of function of peripheral vestibular structures, presenting with chronic dizziness including

Klaus Jahn

klaus.jahn@med.uni-muenchen.de

1 German Center for Vertigo and Balance Disorders (DSGZ), Ludwig-Maximilians University of Munich (LMU), University Hospital Grosshadern, Marchioninistrasse 15, 81377 Munich, Germany

2 Department of Neurology, Schoen Clinic Bad Aibling, Bad Aibling, Germany

3 Clinic for Ear, Nose, Throat and Facial Surgery, Interdisciplinary Center for Vertigo and Neurological Disorders, University of Zurich, Zurich, Switzerland postural imbalance when standing or walking, especially in darkness or on uneven ground. Head or body movements cause visual blurring or oscillopsia, i.e., illusionary bouncing of the visual scene, which causes difficulties in gaze stabilization and keeping one's balance [23]. In most patients, this results in a lower quality of life and a higher risk of falls [24]. The only therapeutic option currently available to sufficiently improve outcome for BVP patients is vestibular rehabilitation therapy (VRT), which aims to improve balance by the training of multisensory postural control to compensate and substitute the vestibular hypofunction [9]. However, the long-term effects of this intervention are limited [21].

Recently, white noise galvanic vestibular stimulation (nGVS) at imperceptible levels was used to modify vestibular perception and performance [5, 28]. When an optimal amplitude of noise is added to a nonlinear system, such as the vestibular system, a mechanism known as stochastic 
resonance is hypothesized to enhance the ability to detect and process weak signals [19] and hence to improve vestibular functions [16]. nGVS transcutaneously delivered to the mastoid processes has been shown to facilitate postural stabilization while standing or walking in healthy subjects as well as in patients with BVP $[8,29,30]$. This makes it a promising non-invasive treatment option for patients with peripheral vestibular hypofunction.

It is not known whether nGVS might induce beneficial synergistic effects when combined with VRT in patients with BVP. Hence, the primary goal of this study was to determine whether the application of nGVS during VRT promotes a better overall recovery than rehabilitation alone (i.e., during sham nGVS) in patients with BVP.

\section{Methods}

This double-blinded clinical explorative study aimed to evaluate the impact of imperceptible amounts of nGVS on the efficacy of vestibular rehabilitation in patients with BVP. The study protocol was approved by the ethics committee of the University of Munich and was conducted in accordance with the Declaration of Helsinki. All participants gave their written informed consent.

\section{Subjects}

Twenty-three patients (9 females, mean age $62.3 \pm 14.3$ years) participated in this study. All of them showed a clinically proven BVP, confirmed either by bilaterally reduced responsiveness to bithermal $\left(44\right.$ and $30^{\circ} \mathrm{C}$ ) caloric irrigation (mean peak slow-phase velocity $<6 \mathrm{deg} / \mathrm{s}$ ) or a pathological video head impulse test on both sides (gain <0.6) [23].

\section{Noisy galvanic vestibular stimulation}

nGVS was delivered via a pair of conductive-rubber electrodes $(4.0 \mathrm{~cm} \times 6.0 \mathrm{~cm})$, placed in two saline-soaked sponges, that were attached over the left and right mastoid process behind the participant's ears. Electrodes were connected to a portable direct current stimulator (neuroConn ${ }^{\circledR}$, Ilmenau, Germany), which delivered the electrical signal consisting of a zero-mean Gaussian white noise within a frequency range of $0-30 \mathrm{~Hz}$. To identify the individual optimal nGVS amplitude, each patient performed eight quietstanding trials with eyes closed for a duration of $30 \mathrm{~s}$ on a stabilometer platform (Kistler 9261A, Winterthur, Switzerland). During each trial, patients received nGVS at a different intensity (from 0 to $700 \mu \mathrm{A}$ in steps of $100 \mu \mathrm{A}$ ) in a randomized order and their performance during stimulation trials was compared to their baseline performance (i.e., nGVS at
$0 \mu \mathrm{A})$. Improvement in balance performance due to nGVS was determined based on the three different posturography parameters, i.e., mean velocity, area, and root mean square of sway. The stimulation amplitude at which individual patients exhibited the best improvement in all three parameters was assigned as their 'optimal nGVS amplitude'. For the sham condition of nGVS, the intensity of the electrical signal was set to $0 \mu \mathrm{A}$.

\section{Vestibular rehabilitation therapy}

The rehabilitation program was individually adjusted to the deficits and therapeutic demands of each patient, to ensure that VRT exercises were sufficiently challenging to induce treatment effects [26]. The basic exercises included training of gaze stabilization during standing and walking and eye-head coordination during standing and walking as well as practices to optimize balance strategies. To challenge patients even more, exercises could be performed on foam or with eyes closed. Furthermore, several different tasks were combined with each other or performed while walking. These basic exercises were individually adapted to the main demands of each patient. This way, the VRT program specifically targeted the individual physical problems. Each therapy session was guided and supervised by an expert vestibular physical therapist and lasted $30 \mathrm{~min}$.

\section{Procedures}

Participants were randomly assigned to one of two groups. The intervention group received nGVS during VRT; the control group received sham stimulation during VRT. In both trial arms, patients were provided with a VRT program three times a week for 2 consecutive weeks (30 min rehabilitation per session). For patients in the intervention group, nGVS was active during all rehabilitation sessions. Patients in the control group received sham nGVS during each rehabilitation session. Outcome measures were assessed at baseline (T0), after 2 weeks of training (T1) and at 2-week follow-up (T2) (see Fig. 1).

\section{Outcome measures}

Several balance and gait tests and questionnaires were applied at the three assessment time points (T0-T2). Primary outcome was postural stability as assessed during posturography while standing on foam with eyes closed. The amount of body sway was quantified by the mean velocity of sway. Secondary outcomes were the patient's gait performance, mobility, and dynamic balance. Gait assessment was performed on a pressure-sensitive gait mat (GAITRite ${ }^{\circledR}$, CIR System, Sparta, NJ, USA) while walking with eyes closed. Gait performance was evaluated 


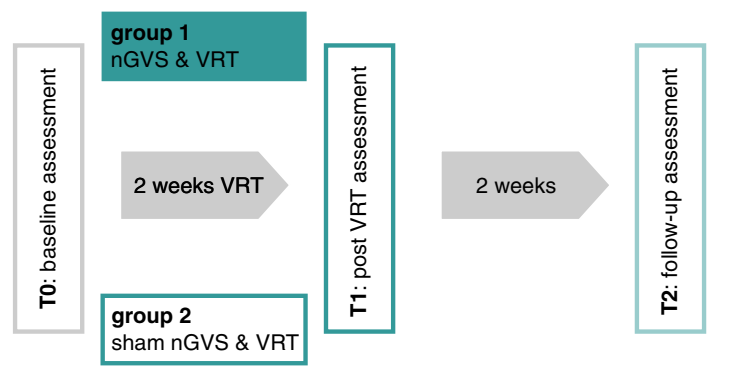

Fig. 1 Scheme of the study protocol. Following an initial baseline assessment, patients were randomly assigned to an intervention group (group 1) or a control group (group 2). Group 1 received 2 weeks of VRT with nGVS at optimal intensity, while group 2 received sham stimulation during training. Treatment effects were assessed immediately after 2 weeks of training (T1) and at 2-week follow-up. $n G V S$ noisy galvanic vestibular stimulation, $V R T$ vestibular rehabilitation therapy

by quantifying walking velocity, base of support, and the coefficient of variation (CV) of stride time. Patients' functional mobility and dynamic balance were assessed by the Berg Balance Scale (BBS; max. 56) [13], the Functional Gait Assessment (FGA; max. 30) [27], and the Timed Up and Go Test (TUG; times of $>13.5 \mathrm{~s}$ are related to an increased risk of falling in older adults) [3]. Additionally, patients completed the following questionnaires: the Dizziness Handicap Inventory (DHI; 16-34 points $=$ mild handicap, $36-52$ points $=$ moderate handicap), $>54$ points $=$ severe handicap) [13] to evaluate the self-perceived handicap due to dizziness, the International Physical Activity Questionnaire (IPAQ; low, moderate, or high physical activity score) [18] to score the level of physical activity, the Falls Efficacy Scale International (FES-I; max. 64) [10] to assess balance confidence and fear of falling, and the Activities-specific Balance Scale (ABC-d; max. 100\%) [13] to assess confidence in performing daily activities.

\section{Statistical analysis}

Data are reported as mean \pm SD. Since most of the outcome measures did not exhibit normal distribution, nonparametric tests were performed to assess differences in treatment effects between the intervention and control groups. For all outcome parameters, the Friedman test was used to assess treatment effects between the three assessment time points (T0, T1, T2). Significant treatment effects were subsequently compared between the intervention and control groups using the Mann-Whitney $U$ test. Results were considered significant if $p<0.05$. Statistical analysis was performed using SPSS (Version 26.0, IBM Corp., USA).
Table 1 Characterization of the study cohorts

\begin{tabular}{llll}
\hline & Intervention group & Control group & \\
\hline Age & $61.92 \pm 15.93 \mathrm{y}$ & $62.64 \pm 13.15 \mathrm{y}$ & $p=0.926$ \\
$\begin{array}{l}\text { Gender } \\
\text { SPV (during }\end{array}$ & $4 \mathrm{f} / 8 \mathrm{~m}$ & $5 \mathrm{f} / 6 \mathrm{~m}$ & $p=0.552$ \\
$\begin{array}{l}\text { caloric irriga- } \\
\text { tion) }\end{array}$ & $5.055 \pm 6.854^{\circ} / \mathrm{s}$ & $5.650 \pm 3.188^{\circ} / \mathrm{s}$ & $p=0.080$ \\
$\begin{array}{l}\text { vHIT gain } \\
\text { Disease duration }\end{array}$ & $0.280 \pm 0.215$ & $0.379 \pm 0.213$ & $p=0.184$ \\
\hline
\end{tabular}

$f$ female, $m$ male, $S P V$ slow-phase velocity, $y$ years

\section{Results}

\section{Characterization of the study cohort}

The study cohort consisted of 23 patients with a clinically proven BVP. The random assignment of these patients to either the intervention group or the control group yielded two homogenous cohorts with respect to age and gender distribution, vestibular hypofunction, and balance deficits (see Tables 1,2). The results of the questionnaires filled by the patients resulted in subjective as well as objective moderate dizziness which did not change significantly during the period of the study (see Table 2).

\section{General effects of VRT}

To examine general effects of VRT, we analyzed training effects on each outcome measure at T1 (after 2 weeks of training) and T2 (at 2-week follow-up) compared to baseline T0. Figure 2 shows in the left panel measurements at baseline (T0), post VRT (T1), and at follow-up (T2) for all participants (sham and intervention group). We did not find VRT-induced changes on postural stability measured by posturography, the BBS scale or any of the balance confidence or physical-activity-related questionnaires. In contrast, we found a moderate training effect regarding patients' gait performance: When walking with closed eyes, the base of support was significantly smaller $(p<0.003)$ at $\mathrm{T} 1$ and returned to baseline level at T2 (see Fig. 2, B, left panel). Comparable improvements were further found for tests of functional mobility, i.e., the TUG $(p<0.013)$ and the FGA $(p<0.040)$ that remained stable at follow-up assessment (see Fig. 2C, D, left panel).

\section{Effects of nGVS}

Identification of optimal nGVS intensity yielded an average nGVS amplitude of $330 \pm 203 \mu \mathrm{A}$. None of the participants felt pain or any other negative symptoms during the application of nGVS. The intervention group received nGVS at 
Table 2 Results of assessments

\begin{tabular}{|c|c|c|c|}
\hline & Intervention group & Control group & \\
\hline T0_-sway velocity (standing on foam, eyes closed) & $313.840 \pm 204.215 \mathrm{~cm} / \mathrm{s}$ & $556.167 \pm 287.200 \mathrm{~cm} / \mathrm{s}$ & $p=0.056$ \\
\hline T1-sway velocity (standing on foam, eyes closed) & $263.421 \pm 166.910 \mathrm{~cm} / \mathrm{s}$ & $501.692 \pm 275.694 \mathrm{~cm} / \mathrm{s}$ & $p=0.023$ \\
\hline T2 - sway velocity (standing on foam, eyes closed) & $332.191 \pm 171.511 \mathrm{~cm} / \mathrm{s}$ & $550.593 \pm 249.715 \mathrm{~cm} / \mathrm{s}$ & $p=0.036$ \\
\hline T0— gait velocity (walking with eyes closed) & $61.833 \pm 26.012 \mathrm{~cm} / \mathrm{s}$ & $63.503 \pm 25.271 \mathrm{~cm} / \mathrm{s}$ & $p=0.712$ \\
\hline T1—gait velocity (walking with eyes closed) & $65.949 \pm 26.640 \mathrm{~cm} / \mathrm{s}$ & $66.919 \pm 22.080 \mathrm{~cm} / \mathrm{s}$ & $p=0.758$ \\
\hline T2-gait velocity (walking with eyes closed) & $61.147 \pm 26.755 \mathrm{~cm} / \mathrm{s}$ & $65.438 \pm 24.439 \mathrm{~cm} / \mathrm{s}$ & $p=0.951$ \\
\hline T0-stride time CV (walking with eyes closed) & $18.240 \pm 13.991 \%$ & $11.580 \pm 7.695 \%$ & $p=0.097$ \\
\hline T1-stride time CV (walking with eyes closed) & $16.129 \pm 19.920 \%$ & $10.532 \pm 7.052 \%$ & $p=0.498$ \\
\hline T2-stride time CV (walking with eyes closed) & $11.350 \pm 5.474 \%$ & $15.126 \pm 13.450 \%$ & $p=1.000$ \\
\hline T0-base of support (walking with eyes closed) & $20.962 \pm 6.117 \mathrm{~cm}$ & $20.417 \pm 7.442 \%$ & $p=0.667$ \\
\hline T1-base of support (walking with eyes closed) & $19.000 \pm 5.791 \mathrm{~cm}$ & $19.625 \pm 6.258 \mathrm{~cm}$ & $p=1.000$ \\
\hline T2-base of support (walking with eyes closed) & $21.556 \pm 5.306 \mathrm{~cm}$ & $20.277 \pm 6.277 \mathrm{~cm}$ & $p=0.389$ \\
\hline T0-TUG & $7.985 \pm 2.676 \mathrm{~s}$ & $6.947 \pm 1.260 \mathrm{~s}$ & $p=0.468$ \\
\hline T1-TUG & $7.450 \pm 2.892 \mathrm{~s}$ & $6.292 \pm 1.287 \mathrm{~s}$ & $p=0.391$ \\
\hline T2-TUG & $7.190 \pm 2.515 \mathrm{~s}$ & $6.412 \pm 0.987 \mathrm{~s}$ & $p=0.356$ \\
\hline T0-FGA & $23.417 \pm 4.963$ & $23.000 \pm 3.194$ & $p=0.805$ \\
\hline T1-FGA & $23.667 \pm 4.030$ & $26.091 \pm 2.737$ & $p=0.137$ \\
\hline T2-FGA & $24.917 \pm 4.166$ & $25.636 \pm 2.942$ & $p=0.877$ \\
\hline T0-DHI & $40.833 \pm 17.837$ & $42.727 \pm 14.867$ & $p=0.734$ \\
\hline T1-DHI & $39.333 \pm 21.309$ & $44.000 \pm 17.550$ & $p=0.423$ \\
\hline $\mathrm{T} 2-\mathrm{DHI}$ & $36.667 \pm 18.884$ & $43.818 \pm 20.851$ & $p=0.423$ \\
\hline T0-BBS & $49.167 \pm 6.645$ & $50.273 \pm 4.268$ & $p=0.951$ \\
\hline $\mathrm{T} 1-\mathrm{BBS}$ & $50.250 \pm 6.369$ & $50.818 \pm 4.750$ & $p=0.975$ \\
\hline $\mathrm{T} 2-\mathrm{BBS}$ & $49.750 \pm 6.152$ & $51.364 \pm 3.982$ & $p=0.733$ \\
\hline $\mathrm{T} 0-\mathrm{ABC}-\mathrm{d}$ & $69.245 \pm 26.027$ & $78.693 \pm 17.255$ & $p=0.372$ \\
\hline $\mathrm{T} 1-\mathrm{ABC}-\mathrm{d}$ & $75.219 \pm 21.443$ & $70.881 \pm 22.502$ & $p=0.601$ \\
\hline $\mathrm{T} 2-\mathrm{ABC}-\mathrm{d}$ & $74.542 \pm 20.264$ & $76.421 \pm 13.406$ & $p=0.902$ \\
\hline T0-FES-I & $25.000 \pm 6.396$ & $25.727 \pm 9.717$ & $p=0.805$ \\
\hline T1-FES-I & $24.083 \pm 6.921$ & $26.364 \pm 9.233$ & $p=0.557$ \\
\hline T2-FES-I & $24.333 \pm 5.662$ & $26.636 \pm 10.957$ & $p=0.902$ \\
\hline
\end{tabular}

$C V$ coefficient of variation, $T U G$ timed up and go test; $F G A$ Functional Gait Assessment, $D H I$ Dizziness handicap inventory, BBS Berg Balance Scale, $A B C$ - $d$ Activities-specific Balance Scale, FES-I Falls Efficacy Scale International

optimal intensities during the complete duration of VRT, while the control group received sham nGVS (i.e., $0 \mu \mathrm{A}$ ) during training. Combining nGVS with VRT did not have any effect on the examined outcome measures after 2 weeks of training or after the 2-week follow-up assessment (see Fig. 2, right panel).

\section{Discussion}

Stochastic electrical vestibular stimulation at imperceptible intensities (i.e., nGVS) has been demonstrated to stabilize static posture and walking performance in patients with BVP $[6,11,22]$. Up to now, vestibular rehabilitation (i.e., VRT) is the only established treatment option for patients with BVP [1]. Here, we applied nGVS in addition to a standardized
VRT treatment in a placebo-controlled double-blinded clinical study to examine whether the combination of both treatments would yield any synergistic effects. While VRT generally induced moderate improvements in patients' balance capabilities, we found no evidence that the combination of nGVS with VRT yields any additional effects on either patients' balance capabilities or their subjective balance selfconfidence. These results will be discussed with respect to (1) the general effects of vestibular rehabilitation training, (2) the absence of any synergistic effects from nGVS, and (3) considerations on whether and how to use both treatment strategies together in the future.

Two weeks of VRT yielded only moderate balance improvements in patients with respect to clinical tests of functional mobility, i.e., the TUG and FGA, and improved gait performance while walking with closed eyes. However, 
A
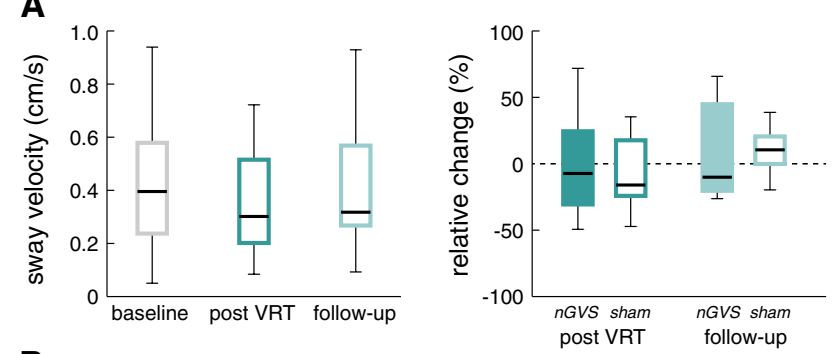

B
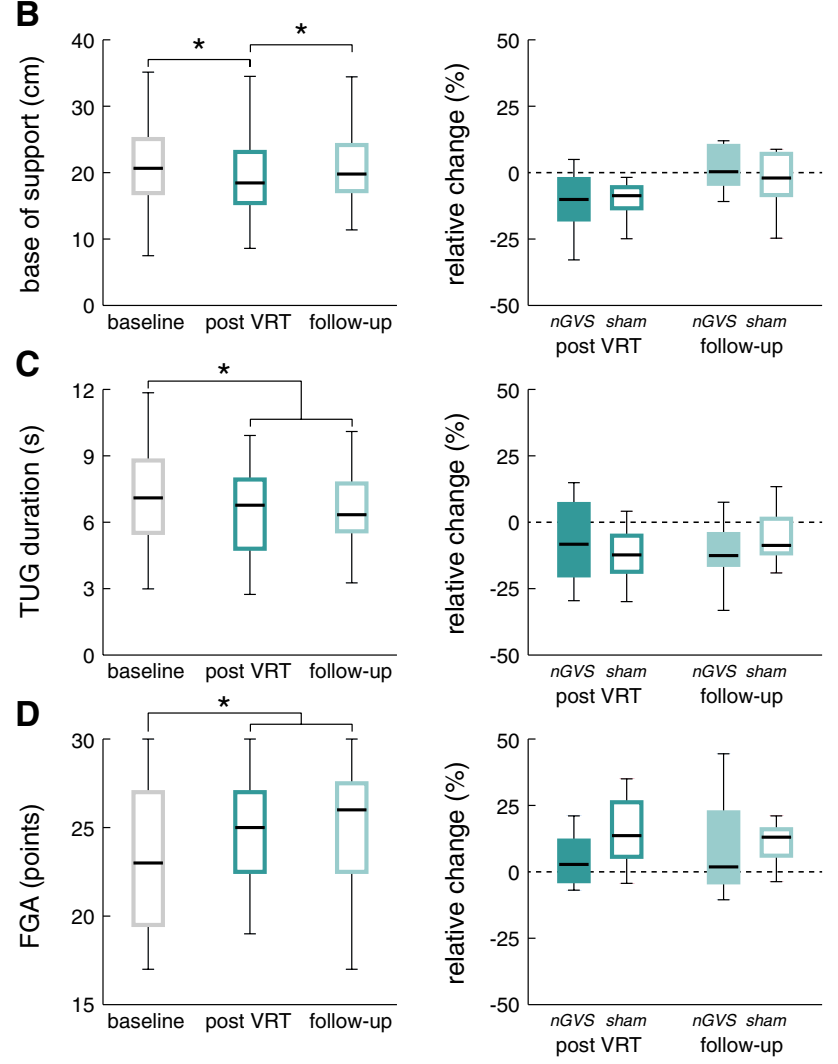

Fig. 2 VRT and nGVS treatment effects. Left panel: general VRTrelated treatment effects (for the combined intervention and control group) at T1 (post VRT) and T2 (follow-up) compared to T0 (baseline assessment) for A sway velocity while standing on foam with eyes closed, B base of support while walking with eyes closed, C TUG, and $\mathbf{D}$ the FGA. Right panel: comparison of relative changes in the respective outcome measures between the intervention and control groups. Moderate general treatment effects of VRT were found for gait capacity, TUG and FGA performance, but not for static posturography. Treatment effects did not differ between the intervention and control groups. VRT vestibular rehabilitation therapy; $n G V S$ noisy galvanic vestibular stimulation, $T U G$ timed up and go test, $F G A$ functional gait assessment; *Indicates a significant difference

no effects were found with respect to clinical scores on balance confidence or static posturography - the primary outcome measure of this study. This observation is in contrast to earlier reports which found clear VRT treatment effects on static body sway and related outcome measures [13]. This discrepancy could be due to differences in the specific VRT protocol and/or differences in study cohorts. For instance,
VRT in the present study only lasted for 2 weeks, while in previous studies, VRT was often applied for considerably longer periods, which might account for treatment effects in BVP $[2,13,17]$. Another reason for the observed weakness of VRT treatment effects could be that patients in our cohort had a relatively moderate severity of BVP-related symptoms compared to previous studies [3] and might therefore only exhibit weak responses when treated with VRT.

Combining nGVS with VRT did not yield any additional effects on patients' stance or gait performance or their balance self-confidence. This absence of synergistic effects of nGVS with VRT might be related to the mode of action of imperceptible stochastic vestibular stimulation, which is thought to improve vestibular function by stochastic resonance, a mechanism in which subthreshold sensory signals become enhanced and detectable by the addition of a particular non-zero amount of noise [4]. Accordingly, nGVS has been shown to particularly improve the perception of weak, subthreshold vestibular cues $[15,16]$ and vestibular-related balance function during absent (i.e., quiet standing [12]) or slow head movements (i.e., slow walking [29, 30]). In contrast, nGVS affected neither vestibular-related perception of suprathreshold cues nor balance function in the presence of dynamic and fast head kinematics in the previous studies. On the other hand, VRT particularly focuses on strong vestibular cues and rapid head movements in order to train deficient vestibular-related balance and ocular-motor functions [25]. Hence, both approaches operate on the opposing ends of the vestibular signal spectrum, which makes them unlikely to exhibit any positive or negative interference effects.

Another reason for the absence of nGVS effects on balance performance and/or confidence could be the point in time at which treatment effects were assessed in this study. Accordingly, assessment of treatment effects at T1 and T2 took place 1 day and 2 weeks after cessation of nGVS, respectively. There is so far no consensus about whether nGVS only acts during ongoing stimulation or whether it exhibits any plastic aftereffects after cessation of stimulation. In favor of the later assumption, Fujimoto et al. reported in an uncontrolled study long-term effects of nGVS on postural stability that lasted up to $6 \mathrm{~h}$ after cessation of stimulation [7]. Later, placebo-controlled studies did not find any evidence for long-term effects of nGVS on vestibular function $[14,20]$. Irrespective of this question, our first assessment of treatment effects took place more than $12 \mathrm{~h}$ after the last application of nGVS and might have thus missed any potential nGVS-induced effects during ongoing stimulation or shortly after stimulation.

The observed absence of any synergistic treatment effects in the combination of VRT with nGVS does, however, not preclude a joint application of both therapeutic strategies for future treatment of BVP. As outlined above, both treatment approaches follow distinct therapeutic principles, target 
rather different sources of deficit in BVP, and act on differing timescales. VRT is usually applied intermittently with the aim to recruit visual and proprioceptive cues to establish a long-term sensory substitution of adaptation for vestibular impairment in BVP, while nGVS only acts during ongoing stimulation with the aim to directly improve the impaired processing of weak vestibular cues in BVP. Hence, both therapeutic options might be suitable partners for a complementary treatment strategy in BVP.

Acknowledgements The authors thank Katie Göttlinger for copyediting the manuscript.

Author contributions $\mathrm{KJ}$, MW, and AK designed the study protocol. $\mathrm{AK}$, TA, and KJ wrote the ethics application and obtained the ethics vote from LMU. JE, SK, TA, and JD recruited patients. JD and KJ supervised the clinical evaluation of the patients. JE, SK, TA, and AK performed the study on patients. VRT was delivered by SK. JE, AK, and MW analyzed the data. JE wrote the first draft of the manuscript. All authors read and approved the final manuscript.

Funding Open Access funding enabled and organized by Projekt DEAL. NeuroConn (Ilmenau, Germany) provided the mobile DCstimulator with the protocol for noisy galvanic vestibular stimulation. The study was supported by the German Federal Ministry for Education and Research (BMBF IFB 01EO1401).

\section{Declarations}

Conflicts of interest KJ received speakers' honoraria from Schwabe Pharma and Rölke Pharma. KJ and MW received funding from NeuroConn (DC-stimulator). On behalf of all authors, the corresponding author states that there is no other conflict of interest.

Ethical approval This double-blinded clinical explorative study aimed to evaluate the impact of imperceptible amounts of nGVS on the efficacy of vestibular rehabilitation in patients with BVP. The study protocol was approved by the ethics committee of the University of Munich and was conducted in accordance with the Declaration of Helsinki.

Informed consent All participants gave their written informed consent.

Open Access This article is licensed under a Creative Commons Attribution 4.0 International License, which permits use, sharing, adaptation, distribution and reproduction in any medium or format, as long as you give appropriate credit to the original author(s) and the source, provide a link to the Creative Commons licence, and indicate if changes were made. The images or other third party material in this article are included in the article's Creative Commons licence, unless indicated otherwise in a credit line to the material. If material is not included in the article's Creative Commons licence and your intended use is not permitted by statutory regulation or exceeds the permitted use, you will need to obtain permission directly from the copyright holder. To view a copy of this licence, visit http://creativecommons.org/licenses/by/4.0/.

\section{References}

1. Alghadir AH, Iqbal ZA, Whitney SL (2013) An update on vestibular physical therapy. J Chin Med Assoc 76:1-8
2. Bae SH, Nam GS, Kwak SH, Kim SH (2021) Importance of highfrequency vestibular function in the prognosis of bilateral vestibulopathy. Clin Exp Otorhinolaryngol 14:192-199

3. Brown KE, Whitney SL, Wrisley DM, Furman JM (2001) Physical therapy outcomes for persons with bilateral vestibular loss. Laryngoscope 111:1812-1817

4. Collins J, Chow CC, Imhoff TT (1995) Stochastic resonance without tuning. Nature 376:236-238

5. Dlugaiczyk J, Wuehr M, Straka H (2020) Electrical stimulation of vestibular endorgans. In: Fritzsch B (ed) The senses: a comprehensive reference, 2nd edn. Elsevier, Oxford, pp 635-671

6. Fujimoto C, Egami N, Kawahara T, Uemura Y, Yamamoto Y, Yamasoba T, Iwasaki S (2018) Noisy galvanic vestibular stimulation sustainably improves posture in bilateral vestibulopathy. Front Neurol 9:900

7. Fujimoto C, Kinoshita M, Kamogashira T, Egami N, Kawahara T, Uemura Y, Yamamoto Y, Yamasoba T, Iwasaki S (2019) Noisy galvanic vestibular stimulation has a greater ameliorating effect on posture in unstable subjects: a feasibility study. Sci Rep 9:17189

8. Fujimoto C, Yamamoto Y, Kamogashira T, Kinoshita M, Egami N, Uemura Y, Togo F, Yamasoba T, Iwasaki S (2016) Noisy galvanic vestibular stimulation induces a sustained improvement in body balance in elderly adults. Sci Rep 6:37575

9. Hall CD, Herdman SJ, Whitney SL, Cass SP, Clendaniel RA, Fife TD, Furman JM, Getchius TS, Goebel JA, Shepard NT, Woodhouse SN (2016) Vestibular rehabilitation for peripheral vestibular hypofunction: an evidence-based clinical practice guideline: from the american physical therapy association neurology section. J Neurol Phys Ther 40:124-155

10. Hill H, McMeekin P, Parry SW (2014) Does the falls efficacy scale international version measure fear of falling: a reassessment of internal validity using a factor analytic approach. Age Ageing 43:559-562

11. Iwasaki S, Fujimoto C, Egami N, Kinoshita M, Togo F, Yamamoto Y, Yamasoba T (2018) Noisy vestibular stimulation increases gait speed in normals and in bilateral vestibulopathy. Brain Stimul 11:709-715

12. Iwasaki S, Yamamoto Y, Togo F, Kinoshita M, Yoshifuji Y, Fujimoto C, Yamasoba T (2014) Noisy vestibular stimulation improves body balance in bilateral vestibulopathy. Neurology 82:969-975

13. Jahn K, Saul AK, Elstner M, Sapa K, Kellerer S (2018) Vestibular rehabilitation therapy and Nintendo Wii balance board training both improve postural control in bilateral vestibulopathy. J Neurol 265:70-73

14. Keywan A, Badarna H, Jahn K, Wuehr M (2020) No evidence for after-effects of noisy galvanic vestibular stimulation on motion perception. Sci Rep 10:2545

15. Keywan A, Jahn K, Wuehr M (2019) Noisy galvanic vestibular stimulation primarily affects otolith-mediated motion perception. Neuroscience 399:161-166

16. Keywan A, Wuehr M, Pradhan C, Jahn K (2018) Noisy galvanic stimulation improves roll-tilt vestibular perception in healthy subjects. Front Neurol 9:83

17. Krebs DE, Gill-Body KM, Parker SW, Ramirez JV, WernickRobinson M (2003) Vestibular rehabilitation: useful but not universally so. Otolaryngol Head Neck Surg 128:240-250

18. Lee PH, Macfarlane DJ, Lam TH, Stewart SM (2011) Validity of the international physical activity questionnaire short form (IPAQ$\mathrm{SF})$ : a systematic review. Int J Behav Nutr Phys Act 8:115

19. Moss F, Ward LM, Sannita WG (2004) Stochastic resonance and sensory information processing: a tutorial and review of application. Clin Neurophysiol 115:267-281

20. Nooristani M, Maheu M, Houde MS, Bacon BA, Champoux F (2019) Questioning the lasting effect of galvanic vestibular stimulation on postural control. PLoS ONE 14:e0224619 
21. Porciuncula F, Johnson CC, Glickman LB (2012) The effect of vestibular rehabilitation on adults with bilateral vestibular hypofunction: a systematic review. J Vestib Res 22:283-298

22. Schniepp R, Boerner JC, Decker J, Jahn K, Brandt T, Wuehr M (2018) Noisy vestibular stimulation improves vestibulospinal function in patients with bilateral vestibulopathy. J Neurol 265:57-62

23. Strupp M, Kim JS, Murofushi T, Straumann D, Jen JC, Rosengren SM, Della Santina CC, Kingma H (2017) Bilateral vestibulopathy: diagnostic criteria consensus document of the classification committee of the barany society. J Vestib Res 27:177-189

24. Ward BK, Agrawal Y, Hoffman HJ, Carey JP, Della Santina CC (2013) Prevalence and impact of bilateral vestibular hypofunction: results from the 2008 US National Health Interview Survey. JAMA Otolaryngol Head Neck Surg 139:803-810

25. Whitney SL, Alghadir AH, Anwer S (2016) Recent evidence about the effectiveness of vestibular rehabilitation. Curr Treat Opt Neurol 18:13
26. Whitney SL, Sparto PJ (2011) Principles of vestibular physical therapy rehabilitation. NeuroRehabilitation 29:157-166

27. Wrisley DM, Marchetti GF, Kuharsky DK, Whitney SL (2004) Reliability, internal consistency, and validity of data obtained with the functional gait assessment. Phys Ther 84:906-918

28. Wuehr M, Decker J, Schniepp R (2017) Noisy galvanic vestibular stimulation: an emerging treatment option for bilateral vestibulopathy. J Neurol 264:81-86

29. Wuehr M, Nusser E, Decker J, Krafczyk S, Straube A, Brandt T, Jahn K, Schniepp R (2016) Noisy vestibular stimulation improves dynamic walking stability in bilateral vestibulopathy. Neurology 86:2196-2202

30. Wuehr M, Nusser E, Krafczyk S, Straube A, Brandt T, Jahn K, Schniepp R (2016) Noise-enhanced vestibular input improves dynamic walking stability in healthy subjects. Brain Stimul 9:109-116 